\title{
Retos para la Unidad Académica de Psicología extensión Jalpa
}

Edith Alejandra Pérez Márquez

\section{Introducción}

$\mathrm{L}$

a Universidad Autónoma de Zacatecas (UAZ) anteriormente era conocida como el Instituto de Ciencias, pero se consolida definitivamente en 1968. Dieciocho años más tarde se funda la Unidad Académica de Psicología (UAP) con apoyo de la Universidad Autónoma de Querétaro (UAQ). Ha tenido varios programas de estudios, sin embargo, el que se desarrolla actualmente fue diseñado en 1997 proponiendo cinco años de formación, divididos en dos años para área básica y tres años en algunas de las siguientes áreas de acentuación que son: social, laboral, clínica y educativa.

La UAP es una de las más grandes para la UAZ con relación al número de alumnos matriculados; en 2007 decide abrir una extensión en Jalpa, la región sur del estado de Zacatecas, que conecta la capital del estado con las ciudades de Guadalajara y Aguascalientes. Existen otras extensiones en Ojocaliente, al este del estado; al norte en Fresnillo y en Juan Aldama. Sin embargo, la extensión Jalpa siempre se ha reconocido por su producción académica en investigación, publicaciones de libros y artículos científicos, así como por alumnos que ganan concursos nacionales de investigación. La región sur del estado de Zacatecas se ha abierto a la aceptación del trabajo psicológico colocando a los egresados de la UAP de Jalpa en puestos de trabajo de gobiernos municipales y del sector privado, atendiendo necesidades de Villanueva, Tabasco, Jalpa, Apozol, Juchipila, Nochistlán de Mejía y Trinidad García de la Cadena, por lo menos.

Uno de los principales problemas a los que nos hemos enfrentado, tanto docentes como alumnos, prestadores de Servicio Social o incluso en Prácticas Académicas y Profesionales, es la perti- nencia de trabajo; hay obstáculos infranqueables que el programa académico no abarca y deja a la intervención psicológica en un papel de incompleta, pues siempre hay que buscar nuevas pautas para continuar trabajando, sobre todo por el compromiso que se ha contraído con la sociedad. Entonces surge la pregunta, ¿cómo se imparte la psicología en las universidades más actualizadas?

\section{Educación Superior Psicológica}

Por los estudios realizados en diversos países es fácil notar que uno de los indicadores de desarrollo a nivel mundial es la educación, sobre todo la universitaria; sin embargo, el presupuesto gubernamental asignado no es suficiente, por lo que las instituciones dependen del subsidio que pueden pagar los alumnos, mismo que en la UAZ es de los más bajos del país. Se sobreentiende que las cuotas de los estudiantes pueden incrementarse, tanto como su calidad lo permita, pues la eficiencia y el costo monetario de la educación van de la mano; en el plano de la realidad cotidiana, precio no es igual 
a eficacia. Para poder hablar de calidad educativa habría que evaluar diversos campos: formación docente, alumnos aceptados, programas (diseño y reestructuración) e infraestructura.

Un estudio realizado por Ocegueda (2014) muestra una comparación entre diferentes países: Corea del Sur, Argentina, Brasil, Chile y México, los últimos cuatro por ser los más desarrollados en Latinoamérica y el primero por ser el número uno en educación a nivel mundial, desbancando a Finlandia. En dicho estudio, los investigadores señalan que México es el menor inversionista en recursos humanos para la educación superior de entre los cuatro países mencionados, mas habría que considerar que es un país de migrantes, tanto en mano de obra barata como en fuga de cerebros.

Esto determina una brecha significativa en la disponibilidad de profesionistas y personal científico, sobre todo de aquéllos que se emplean en actividades de I y D [investigación y desarrollo]. No obstante, la conformación de capacidades científicas va más allá del tamaño del SEs [Sistema de Educación Superior] y requiere apoyarse con políticas específicas para asignar recursos humanos y financieros hacia este tipo de actividades como lo han hecho principalmente los coreanos (Ocegueda, 2014: 183).

Como lo mencionan los autores, no sólo se requiere personal académico que se dedique a la formación de otras personas, sino también que se encarguen del desarrollo de investigaciones y la creación de patentes de todas las áreas científicas. México cuenta con el material creativo suficiente para la solución de problemas, en comparación con los mejores a nivel mundial, pero un docente investigador que tiene una carga laboral repleta de clases, como sucede en la mayoría de los casos en las universidades en México, no tiene las herramientas para innovar, ni el tiempo, ni la actualización, ni la calidad de vida para llevar a cabo dicha tarea.

De acuerdo con Ocegueda (2014) hay tres indicadores de calidad para las Instituciones de Educación Superior (IES) que son: actividades académicas del profesor dentro de la institución, formación académica y tamaño de los grupos; para la extensión Jalpa de la UAP el primer punto se traduce en clases, asesorías de tesis fuera del horario de trabajo, tutorías mensuales y otras actividades extracurriculares pero académicas, tales como cursos propedéuticos, Semana de Psicología como festejo de cada aniversario de la fundación de la UAP, convocatoria de admisión, celebración del día del Estudiante y del día del Psicólogo y graduaciones cada ciclo escolar. En todas las actividades anteriores no participa toda la planta docente, lo que se traduce en exceso de trabajo para unos cuantos.

Las actividades académicas de la extensión Jalpa de la UAP de la UAZ son eficientes y reconocidas por directores de Unidades Académicas de la universidad, ya que durante el ciclo escolar se llevan a cabo talleres de profesores de la institución y de invitados, presentaciones de libros escritos por docentes de la misma escuela, conferencias, cursos extracurriculares, participación en cátedras nacionales, ponencias en congresos nacionales e internacionales, mesas de trabajo, participación en asamblea ciudadana de la región, estancias docentes, movilidad académica, entre otras que contribuyen de gran manera a la formación de nuestros estudiantes.

La formación docente es actualizada y oportuna, pues de los once profesores para la extensión Jalpa, tres son doctores, tres doctorantes y cinco maestros, de los cuales dos son miembros del Sistema Nacional de Investigadores (SNI) y cinco con nombramiento del Programa para el Desarrollo Profesional Docente (PRODEP), algunos más en proceso de certificación del Consejo Nacional de Normalización y Certificación de Competencias Laborales (CONOCER-SEP), todos realizamos publicaciones en revistas indexadas con sobresaliente calidad, incluso se ha considerado que lo único que falta es tiempo para seguir formándose, hacer investigación y registrarla.

De acuerdo con el Qs World University Rankings (2016) hay diez universidades alrededor del mundo que cubren las necesidades de calidad educativa para estudiar una Licenciatura en Psicología, tomaremos dos casos para revisar, en América del Norte es la Universidad de Harvard (2016), mientras que para Europa, la Universidad de Cambridge (2016). El programa del Departamento de Psicología en la Universidad de Harvard consta de cuatro áreas: ciencias clínicas, de desarrollo, social y sobre la cognición, el cerebro y el comportamiento. Los miembros de cada área, tanto alumnos como docentes, son invitados a conocer más de una para no tener una visión cerrada de ellas; así mismo 
participan en diversas investigaciones del Departamento de Psicología.

Por otra parte, la Universidad de Cambridge (2016) ha fusionado el Departamento de Psicología Experimental con el de Psicología Social y Desarrollo y con el Centro de Investigación Familiar, por lo que una de sus características es ser multidisciplinario, lo que coloca a sus estudios en psicología en la tercera posición de la clasificación mundial, de acuerdo con la Qs World University Rankings (2016), y reconocida por la Asociación Británica de Psicología. Su laboratorio de psicología inició funciones en 1912, por lo que nos lleva más de 100 años de ventaja, ya que que en la UAP de la UAZ todavía no comienza a funcionar.

La formación inicial en psicología en Zacatecas debe basarse en el Modelo UAZ Siglo XXI (2005), mismo que propone englobar valores, conocimientos, habilidades y cultura; es humanista, pues atiende a grupos vulnerables y tiene una visión de la realidad social para construir un mundo mejor, no sólo para sí mismos sino para las nuevas generaciones; tiene un enfoque científico y tecnológico, con miras a ser sustentable; los valores más importan- tes son la tolerancia y responsabilidad; los docentes son líderes del currículo que ofrece programas de calidad educativa centrados en el aprendizaje, pero sobre todo aplicables en distintos planos de realidad, promoviendo la acción y reflexión crítica para dar respuesta a los retos del mundo actual, sus puntos de vista son vigentes ya que las actualizaciones son entre los dos y cinco años como máximo.

De acuerdo con los parámetros establecidos por el Consejo Nacional para la Enseñanza e Investigación en Psicología (CNEIP), para el cumplimiento de la evaluación que ha acreditado a la UAP se debe cambiar el plan de trabajo curricular de la licenciatura, por lo que se ha trabajado en ello durante los últimos años a través de equipos en las siguientes áreas: Básica, Clínica, Educativa, Laboral y Social, a las que se les han realizado diversas observaciones de actualización, por lo que se ha optado por implementar un plan curricular por medio de Unidades Didácticas (udis) y de movilidad para las áreas en las que el alumno deberá seleccionar dos materias, pero con la opción de dos materias de otras áreas, con asesoría de un tutor, para acceder a una formación psicológica completa.
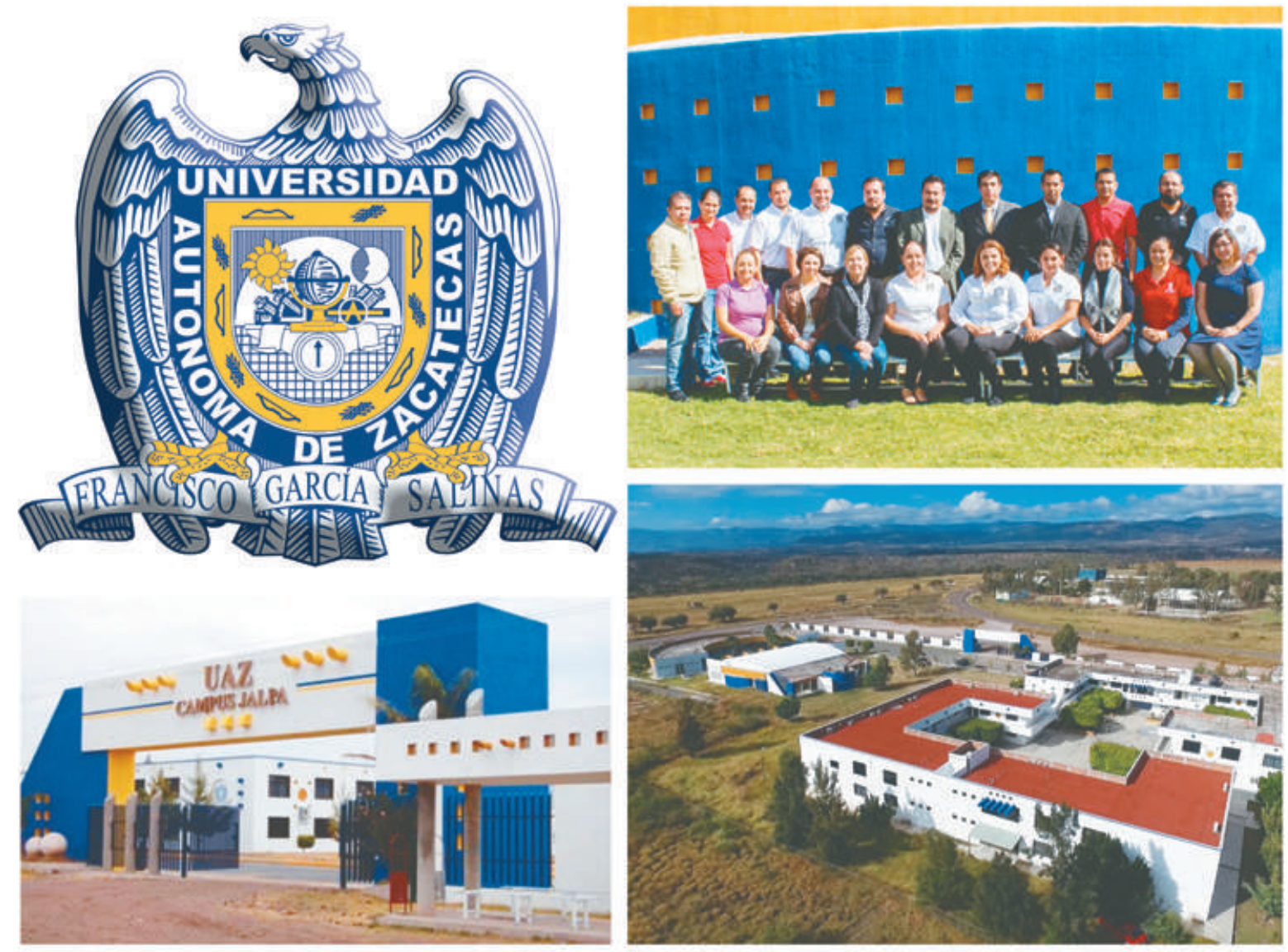
《 No sólo se requiere personal académico que se dedique a la formación de otras personas, sino también que se encarguen del desarrollo de investigaciones y la creación de patentes de todas las áreas científicas 》〉

\section{Conclusión}

La situación académica actual de Psicología en la UAZ está marcada por un plan curricular obsoleto, que ya no cubre las necesidades reales de los empleadores, sobre todo de empresas, secretarías gubernamentales o incluso clientes individuales. Urge actualización en la preparación de los estudiantes, ya que el plan de estudios data de 1997, mientras que el Modelo UAz Siglo XXI se formó ocho años después, por lo que ya tendría que haberse revisado para alguna reestructuración curricular por lo menos dos veces, considerando que fuera cada cinco años, o cinco veces, si fuera cada dos años.

Hay calidad de trabajo porque los docentes, por lo menos de la UAP, se han actualizado de manera continua pero individual, cada uno ha buscado su propio desarrollo intelectual con doctorados en el país y en el extranjero, maestrías, certificaciones y otros estudios; aunque la mayoría migran a realizar estudios en universidades españolas, que no tienen malas puntuaciones pero tampoco están entre las mejores del mundo, como es el caso mencionado de la Universidad de Harvard (2016) o la Universidad de Cambridge (2016); aun así, los docentes e investigadores de universidades en España han comentado que el programa de Psicología UAZ es obsoleto, sobre todo en el área clínica.

Según las observaciones que realizó CNEIP en la actualización del plan de estudios para la certificación de la UAP, las áreas educativa y social son las más pertinentes y sólo se les realizaron un par de correcciones; tomando en cuenta las características generales de las universidades extranjeras mencionadas, las neurociencias, los estudios del desarrollo y la psicología social son las más oportunas para la psicología actual. Los métodos psicológicos para una sociedad en pleno cambio, por su entrada al nuevo milenio, requieren evolucionar.

Si la UAZ deja a un lado cuestiones políticas, la sociedad en general, a nivel nacional, podría darse cuenta de la calidad que ofrece, que es mayor al costo que cobra a los alumnos que ingresan, así podría formarse también una identidad universitaria que lleva el lema institucional que ahora le rige: "El nuevo rostro del orgullo universitario", pues es un organismo que podría estar produciendo sus propias patentes y resolviendo así los problemas económicos a los que se enfrenta actualmente, mismos que dañan tanto física como moralmente a todos sus miembros, desde directivos, personal administrativo, hasta docentes y estudiantes.

De igual manera, en el caso de la extensión Jalpa de la UAP de la UAZ, el entusiasmo no aminora, pues también hay estudiantes comprometidos que buscan actualizarse y trabajar en diversas áreas de la psicología desde su área de formación, ampliando su campo de conocimientos aunado con las actualizaciones docentes.

El tiempo que se requiere para poder realizar investigaciones, lograr los registros de las mismas y tener estudios superiores a los alcanzados actualmente, son retos a los que el equipo de trabajo, conformado por once profesores, tendremos que afrontar, pues como coloquialmente se dice "hay más tiempo que vida".

\section{Fuentes de consulta}

Harvard University (2016). Department of Psychology: Overview. Massachusetts, Eu. Recuperado el 20 de diciembre de 2016, en: http:// bit.ly/2nnZ21f

Ocegueda, J., Miramontes, M. y Moctezuma, P. (2014). La educación superior en México: un estudio comparativo. Ciencia Ergo Sum, pp. 181-192.

Qs World University Rankings (2016). Educate Every Child on the Planet: The World Top '20 Project. EUA. Recuperado el 12 de diciembre de 2016, en: www.worldtop20.org/ Universidad Autónoma de Zacatecas (2005). Modelo UAZ Siglo XXI. Zacatecas: UAZ

University of Cambridge (2016). Department of Psychology: About us. Cambridge, Inglaterra, Reino Unido. Recuperado el 16 de diciembre de 2016, en: http://www.psychol. cam.ac.uk/about-us 